\title{
Intra-articular long head of the biceps tendon: magnetic resonance-arthrography classification and review of literature
}

\author{
Nicola Tarallo ${ }^{1 \mathrm{~A}}$, Maria C. Morgano ${ }^{1 \mathrm{~B}}$, Marco Curti ${ }^{1 \mathrm{~B}}$, Emanuela Spanò ${ }^{1 \mathrm{~B}}$, Alessandro Castagna ${ }^{2 \mathrm{~B}}$, \\ Eugenio A. Genovese ${ }^{1,2,3 \mathrm{~A}}$ \\ ${ }^{1}$ The Circolo Hospital and Macchi Foundation, University of Insubria, Varese, Italy \\ ${ }^{2}$ Orthopedics of the Shoulder and Elbow Unit, Rozzano Hospital, Milan, Italy \\ ${ }^{3}$ Medical Clinical Center Intermedica-Columbus, Milan, Italy
}

\section{Abstract}

Purpose: Anatomical variants of the long head of the biceps (LHB) and diseases of the rotator interval structures may contribute to shoulder instability. The rotator interval and the LHB tendon are closely associated anatomic structures that confer stability to the shoulder. Anatomical variants around the origins of the long head of the biceps (LHB) are reported to occur with a frequency of 1.9-7.4\%. In the past years, many authors have proposed different approaches for the identification and characterization of LHB and rotators interval. Magnetic resonance (MR) arthrography is considered the reference standard in imaging to diagnose superior shoulder diseases. However, few authors have analysed the anatomical variants and the relation between those and shoulder instability. This study aimed to identify the frequency of variants observed during arthroscopic shoulder surgeries, and to classify them based on the Dierickx classification system.

Material and methods: In $326 \mathrm{MR}$ arthrograms we investigated the incidence of LHB anatomical variations and their association with shoulder diseases.

Results: We found 252/326 (77.3\%) cases of LHB free, 40/326(12.26\%) cases of LHB adherent, 31/326(9.50\%) cases of mesotenon, and $3 / 326(0.9 \%)$ cases of split biceps. The prevalence of rotator interval synovitis in the mesotenon group was greater than in the LHB-free group. Moreover, in the LHB-adherent group we observed increased incidence of sublabral recess and SLAP lesions compared with the LHB-free group.

Conclusions: MR-arthrography is useful in the evaluation of superior shoulder structures. A relationship exists between LHB anomalies and superior shoulder instability.

Key words: shoulder, anatomical variants, SLAP lesions, instability shoulder, long head biceps, MR arthrography.

\section{Introduction}

Anatomical variants of the long head biceps (LHB) and diseases of the structures of the rotator interval may contribute to superior shoulder instability, and these conditions have been the subject of interest both for shoulder surgeons and radiologists [1-4]. The rotator interval and the LHB tendon are closely associated anatomic structures that confer stability to the shoulder $[1,5,6]$. Anatomical variants around the origins of the LHB are reported to oc- cur with a frequency of $1.9-7.4 \%$, with many variations in shape, including complete absence, split or Y-shaped variant, and extracapsular origin of LHB, as reported by Jeong et al. [7]. In the past years, many authors have proposed different approaches for the identification and characterization of LHB and rotator interval [5,6]. Clinical and arthroscopic diagnoses of rotator interval abnormalities are sometimes difficult, and imaging nowadays represents the best approach to achieve a reliable diagnosis. In particular, magnetic resonance (MR) arthrography is considered

\section{Correspondence address:}

Dr. Nicola Tarallo, The Circolo Hospital and Macchi Foundation, University of Insubria, Viale Luigi Borri, 57, Varese, Lombardy, Italy, e-mail: tarallo.nicola@gmail.com Authors' contribution:

A Study design · B Data collection · C Statistical analysis · D Data interpretation · E Manuscript preparation · F Literature search · G Funds collection 
the reference standard in imaging to diagnose superior shoulder diseases $[8,9]$. However, few authors have systematically classified or analysed the variants with a large group of participants. Moreover, the relationship between the variants and shoulder joint diseases is controversial [10-15]. This study aimed to identify the frequency of variants observed during arthroscopic shoulder surgeries, to classify them based on the Dierickx classification system (Table 1) [16]. Furthermore, we correlated these anatomical variants with some lesions found in superior shoulder diseases.

\section{Material and methods}

\section{Patients}

We retrospectively reviewed 482 consecutive MR shoulder arthrographies. A total of 156 tests were excluded from the study: the supraspinatus (SSP) tendon complete lesion (91 tests), previous surgery (33 tests), motion artifacts or inadequate joint distension (22 tests), adhesive capsulitis (6 tests), and LHB tendon complete lesion (4 tests).

The remaining $326 \mathrm{MR}$ arthrographies were performed in 317 patients, (218 males and 99 females, mean age 46.5 years, age range $18-73$ years); 187 right shoulders and 139 left shoulders were examined.

The LHB anatomical variants of the intra-articular portion course were considered and classified according to Dierickx's arthroscopic classification [16] (Table 2), based on the relationship with the SSP tendon [17]. Moreover, in the analysis, superior shoulder associated diseases and superior shoulder anatomical variants were assessed.

\section{Magnetic resonance arthrography imaging}

Three 1.5-Tesla MR imaging systems (Eclipse, PickerMarconi [Unit 1], Magnetom Avanto, Siemens [Unit 2], Achieva XR, Philips [Unit 3]) were used with a dedicated shoulder array coil. The patient was lying on the resonance table in a supine position and the shoulder was imaged in a neutral position with the arm flanking the body and the thumb pointing up. All patients were asked to give written informed consent before the procedure. MR arthrography was performed with the injection of $20 \mathrm{ml}$ of paramagnetic contrast agent (Magnevist $2 \mathrm{mmol} / \mathrm{l}$, Bayer-Schering; Dotarem $2.5 \mathrm{mmol} / \mathrm{l}$, Guerbet), through a $20 \mathrm{G}$ needle previously positioned, without ultrasound guidance, inferolaterally to the coracoid process, thus reaching the anteromedial profile of the humeral head. The examination was performed within 30 minutes of injection of contrast agent. MR arthrographies were performed according to the following protocols.

Unit 1:

- multiplanar (coronal, axial and sagittal plane) T1weighted spin-echo sequences: repetition time (RT): $500 \mathrm{~ms}$, echo time (ET): $12 \mathrm{~ms}$, matrix $256 \times 512$ pixels, $0.8 \times 0.8 \mathrm{~mm}$ pixel size, number of signals acquired (NSA): 1, thickness: $3.5-4 \mathrm{~mm}$;

- coronal fat-saturated proton density (PD) and T2weighted fast spin-echo sequences (FSE PD/T2 FAT SAT): RT: $4000 \mathrm{~ms}$, ET: 19/96 ms, flip angle [FA]: $90^{\circ}$, matrix: $256 \times 256$ pixels, NSA: 2 , thickness: $4 \mathrm{~mm}$;

- axial fat-saturated T1-weighted fast spin-echo sequences with isotropic voxel (T1 FSE FAT-SAT): RT: $31 \mathrm{~ms}$, ET $7 \mathrm{~ms}$, FA: $90^{\circ}$, matrix $256 \times 256$ pixels, $0.8 \times 0.8 \mathrm{~mm}$ pixel size, NSA: 2 , thickness: $0.5 \mathrm{~mm}$.

Unit 2:

- axial fat-saturated T1-weighted fast spin-echo sequences with isotropic voxel (T1 FSE FAT-SAT): RT $7.5 \mathrm{~ms}$, ET: $2.64 \mathrm{~ms}$, FA: $12^{\circ}$, matrix $320 \times 307$ pixels, $0.8 \times 0.8 \mathrm{~mm}$ pixel size, NSA: 1 , thickness: $0.7 \mathrm{~mm}$;

- oblique coronal (parallel to the long axis of the SSP tendon) and sagittal (perpendicular to the long axis of the SSP tendon) T1-weighted turbo spin-echo sequences (TSE T1); RT: $400 \mathrm{~ms}$, ET: $11 \mathrm{~ms}, \mathrm{FA}: 90^{\circ}$, matrix $384 \times 307$ pixels, 0.8 $\times 0.8 \mathrm{~mm}$ pixel size, NSA: 1 , thickness: $3.5-4 \mathrm{~mm}$;

- coronal fat saturated PD/T2-weighted fast spin-echo sequences (FSE PD/T2 FAT SAT): RT $3300 \mathrm{~ms}$, ET 12/ $106 \mathrm{~ms}$, FA: $150^{\circ}$, matrix $230 \times 256$ pixels, $0.8 \times 0.8 \mathrm{~mm}$ pixel size, NSA: 1, thickness: $3.5-4 \mathrm{~mm}$.

Table 1. Dierickx's arthroscopic classification [11]

\begin{tabular}{|l|l|l|}
\hline MESO & $\begin{array}{l}\text { The "mesotenon" family contains } \\
5 \text { types of connections that allow } \\
\text { very good movement between } \\
\text { the LHB and the rotator cuff. }\end{array}$ & $\begin{array}{l}\text { MESO-VI (vinculum) } \\
\text { MESO-SB (small band) } \\
\text { MESO-PU (pulley-like sling) } \\
\text { MESO-PA (partial mesotenon) } \\
\text { MESO-CO (complete mesotenon) }\end{array}$ \\
\hline ADH & $\begin{array}{l}\text { The'adherent' family contains 4 types } \\
\text { of stronger connections between } \\
\text { a single LHB tendon and the capsule. }\end{array}$ & $\begin{array}{l}\text { ADH-PM (partially medially adherent to the SSP) ADH-PL (partially laterally adherent to the SSP) } \\
\text { ADH-CL (complete adherent; attaching to the labrum) } \\
\text { ADH-CO (complete adherent to SSP; not attaching to the labrum) }\end{array}$ \\
\hline SPL & $\begin{array}{l}\text { The 'split' family contains the 2 types } \\
\text { of split biceps. }\end{array}$ & $\begin{array}{l}\text { SPL-DO (split biceps double origin) } \\
\text { SPL-RE (split biceps reversed type) }\end{array}$ \\
\hline ABS & $\begin{array}{l}\text { Indicates patients with a complete } \\
\text { absence of the LHB }\end{array}$ & \begin{tabular}{l} 
ABS (complete absence of LHB) \\
\hline
\end{tabular}
\end{tabular}

MESO - mesotenon, ADH - adherent, SPL - split, ABS - absence, LHB - long head biceps 
Table 2. Variations of the IA long head biceps. Image obtained from [16]

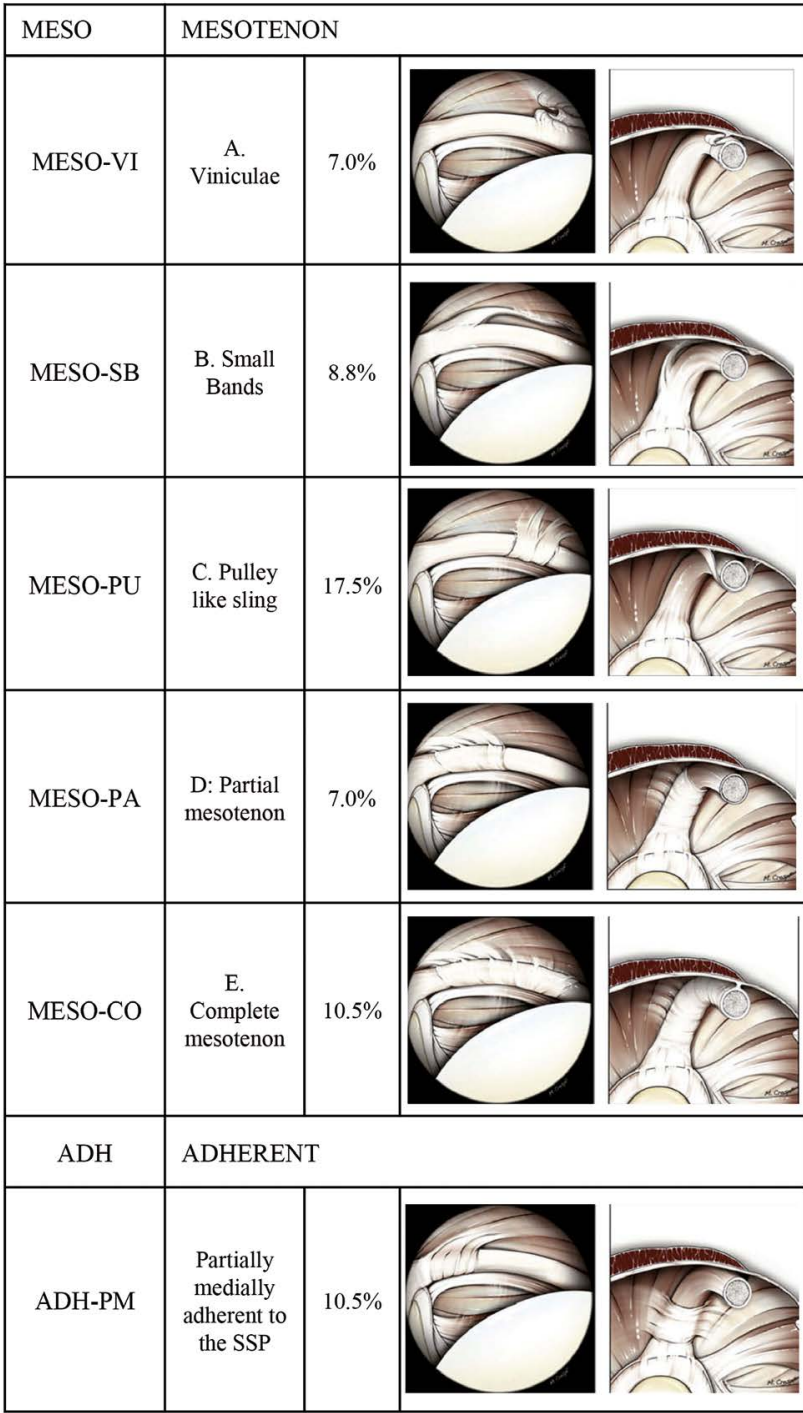

Unit 3:

- axial T1-weighted sequences with isotropic voxel: RT $9.5 \mathrm{~ms}, \mathrm{ET}: 4.7 \mathrm{~ms}, \mathrm{FA}: 7^{\circ}$, matrix $320 \times 307$ pixels, $0.8 \times$ $0.8 \mathrm{~mm}$ pixel size, NSA: 1 , thickness: $0.54 \mathrm{~mm}$;

- oblique coronal and sagittal T1-weighted turbo spinecho sequences (TSE T1): RT: $500 \mathrm{~ms}$, ET: $18 \mathrm{~ms}$, FA: 90 matrix $384 \times 307$ pixels, $0.8 \times 0.8 \mathrm{~mm}$ pixel size, NSA: 1 , thickness: $3.5-4 \mathrm{~mm}$;

- coronal fat-saturated PD/T2-weighted (dual) fast spinecho sequences (FSE PD/T2 FAT SAT): RT 4000 ms, ET: 10/80 ms, FA: $90^{\circ}$, matrix $230 \times 256$ pixels, $0.8 \times 0.8 \mathrm{~mm}$ pixel size, NSA: 1, thickness: $3.5-4 \mathrm{~mm}$.

The field of view (FOV) was variable from 16 to $20 \mathrm{~cm}$.

\section{Statistical analysis}

Fisher's exact test (for non-parametric statistics analysis) was used to find correlation with LHB pulley anatomic variations and superior shoulder-associated pathology. A $p$ value $<0.05$ was considered to indicate a significant difference.

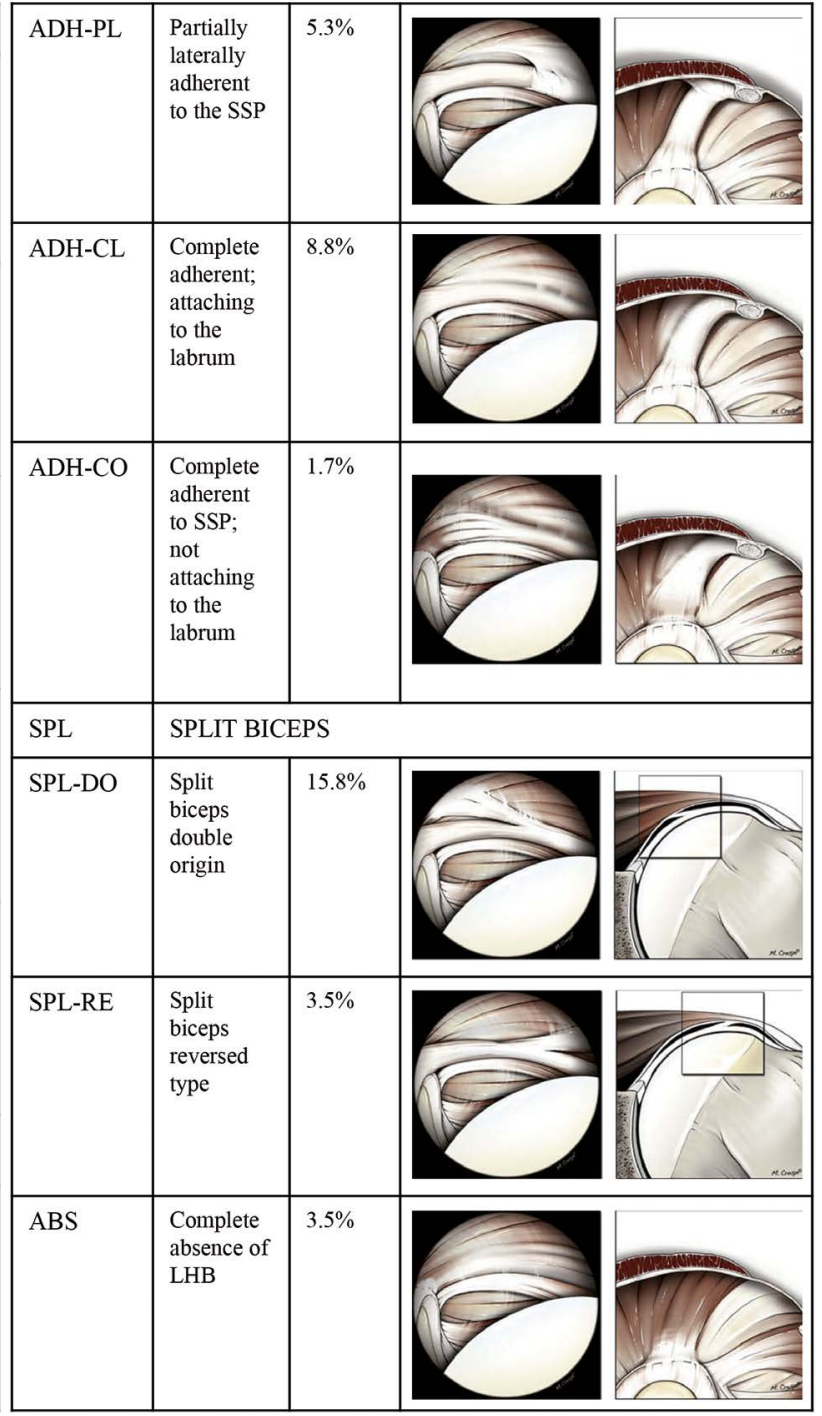

\section{Results}

\section{LHB intra-articular portion course}

The LHB intra-articular portion course prevalence is summarized in Table III. In brief, in 252/326 (77.3\%) cases we found the LHB-free variant (Figure 1). The LHB adherent and LHB mesotenon were found in $12.26 \%$ and $9.50 \%$ of cases, respectively, whereas the LHB split divisum was found in only $0.92 \%$ of cases $(n=3)$; no LBH-absent cases were found (Figures 2-5 show these anatomical configurations). Variation in attachment configuration and in thickness or shape of LHB is of potential significance to labral injures of the superior glenoid labrum (Figures 6 and 7).

\section{Associated superior shoulder diseases}

For the purpose of this study only the superior shoulder diseases involving the glenoid portion covered between 9 o'clock and 3 o'clock were considered. In our series only $112 / 326(34.3 \%)$ of shoulders did not show superior 


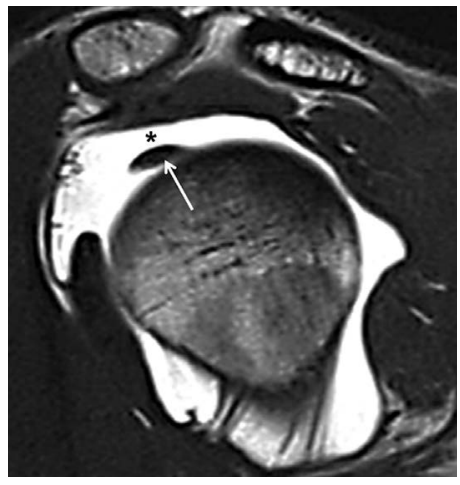

Figure 1. Magnetic resonance arthrography, SE T1w image on oblique sagittal plane. Normal course of long head biceps (LHB) tendon (arrow) that seems to be free in intra-articular position, contrast agent is located between LHB tendon and supraspinatus tendon (asterisk)

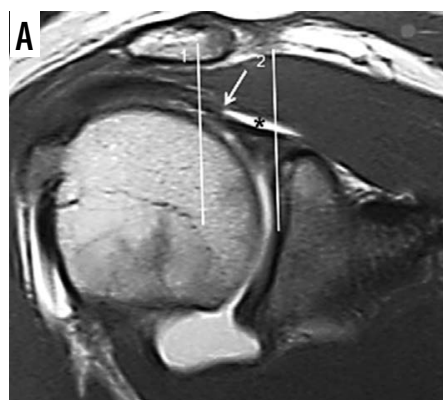

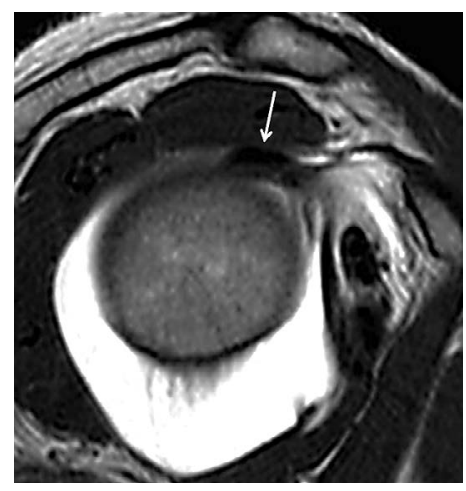

Figure 2. Magnetic resonance arthrography. SE T1w image on oblique sagittal plane. ADH-CL anatomic variation, long head biceps tendon is adherent to supraspinatus tendon, without contrast agent between these tendons (arrow)

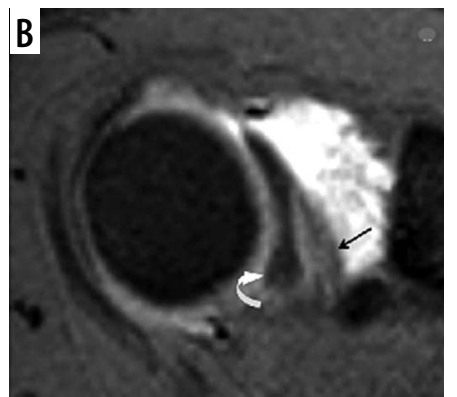

Figure 3. Magnetic resonance arthrography. A) SE T1w image on oblique coronal plane, through sections 1 and 2. ADH-PL anatomic variation, long head biceps (LHB) is adherent to supraspinatus tendon only in its lateral portion (arrow), while on medial portion there is agent contrast between LHB and supraspinatus tendon (asterisk). B) T1w FAT SAT image with isotropic voxel on axial plane. SPL-D0 anatomic variation, LHB has double origin from supraspinatus tendon (curved arrow) and from glenoid (black arrow)
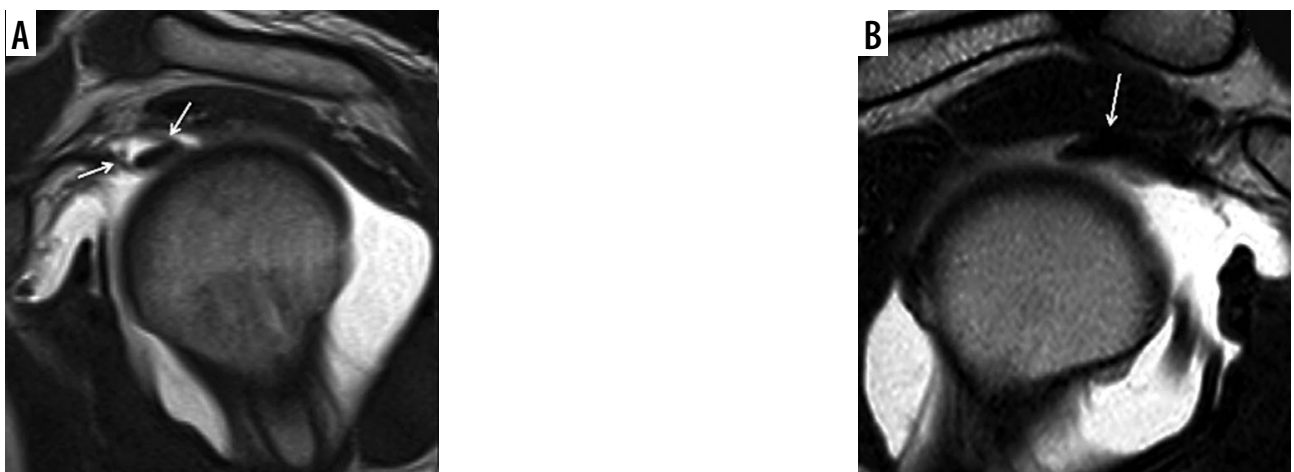

Figure 4. Magnetic resonance arthrography. SE T1w image on oblique sagittal plane. A) MESO-PU anatomical variant, thin intra-articular hypointense hammock-like sling around the long head biceps (LHB) tendon (arrows). B) MESO-PA anatomical variant, LHB tendon is partially attached to inferior surface of supraspinatus tendon (arrow), contrast agent forms an obtuse angle with the anterior portion of the LHB tendon and an acute angle with the posterior portion
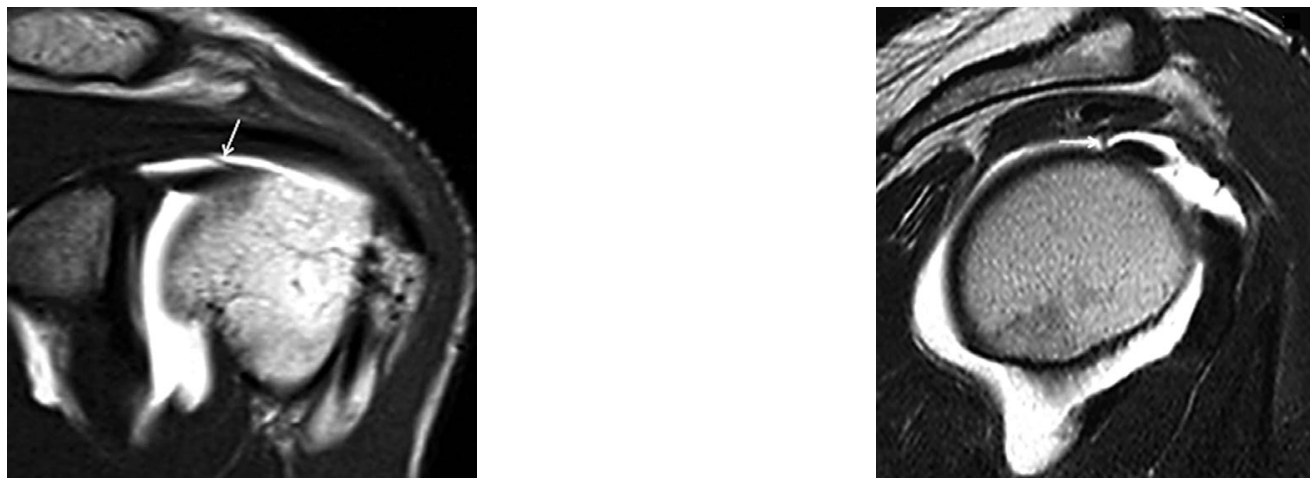

Figure 5. Magnetic resonance arthrography. A) SE T1w image on oblique coronal plane. MESO-SB anatomical variant with thin intra-articular hypointense synovial band (arrow), which from medial to lateral connects the rotator cuff with the biceps. B) SE T1w image on oblique sagittal plane. MESO-VI anatomical variant, biceps is connected to the rotator cuff through an hypointense intra-articular fine string with vertical course (arrow) 
shoulder diseases. In the remaining cases, $81 / 214$ (37.8\%), there were many diseases associated at the same time, resulting in 256 diseases in total.

\section{Superior shoulder anatomical variants}

A total of 97 anatomic variants, found in 89 shoulders, had associated together in 10/89 (11.2\%) cases. The most prevalent conditions were hypoplasia or agenesis of the glenoid labrum without MGHL hypertrophy in 30/326 (9.2\%) cases, and sublabral foramen that was diagnosed in $31 / 326(9.5 \%)$ patients.

\section{Analysis of results}

We evaluated the presence of anatomic variations of LHB tendon intra-articular portion course, superior shoulder diseases, and superior shoulder anatomic variations. The data were also tested using a Fisher test. We found statistically significant values in the comparison between the mesotenon group and the LHB-free group as regards the presence of rotator interval synovitis $(p=0.007)$ and between the LHB adherent group and the LHB-free group both as regards the SLAP lesions $(p=0.05)$, both as regards the presence of sub-labral recesses $(p=0.018)$.

\section{Discussion}

Superior instability is an important cause of chronic pain, and it is difficult to diagnose with a physical examination only, so the support of imaging is fundamental. Superior instability is frequently associated with different anatomical variants or pathological conditions. Many classifications of shoulder instability exist. Among them, the most commonly adopted by orthopaedic surgeons is the Matsen-Snyder-Castagna classification, which includes traumatic unidirectional instability with Bankart lesion requiring surgery (TUBS), a-traumatic multidirectional bilateral instability responsive to rehabilitation, inferior capsular shift and interval closure (AMBRII), and a group of subtle conditions of minor instability secondary to repeated microtraumas, known as acquired instability from overstress, which is usually treated with surgery (AIOS). The labral position is located by superimposing the face of a clock onto the surface of the glenoid, and by convention 12 oclock is superior and 6 o'clock is inferior; the 9 to 3 oclock position of the glenoid fossa corresponds to the superior zone of the glenoid labrum or supraequatorial area, 3 o'clock is the anterior portion and 9 o'clock the posterior portion. The supraequatorial region includes structures that play an important role in static and dynamic stabilisation of the glenohumeral joint (rotator interval, long head of the biceps brachii, superior glenoid labrum, superior and middle glenohumeral ligaments and tendons of rotator cuff). Lesions or anatomical variants of these structures are related to shoulder instability.
The commonest causes of shoulder pain are abnormalities of the rotator interval or LHB tendon, and SLAP lesions [8].

Moreover, variation in attachment configuration of LHB is of potential significance to labral injuries of the superior glenoid labrum (Figure 8). According to Jakanani et al. [1], variation in the anatomy of the biceps origin influences the type of labral tears that occur in patients with shoulder instability. In particular, they found a significant association between the predominantly posterior LHB attachment and SLAP. For example, through an interesting theory, they speculated that a predominantly posterior tendon attachment predisposes to a SLAP tear, as reported (Figure 8). Superior shoulder diseases and anatomic variations have been investigated in several studies $[2,3,16]$. Rotator interval abnormalities were also called "hidden" injuries by Walch et al. [18], referring to the difficulty of the arthroscopic identification, which is probably due to the anterior portal where the usual lax appearance of the anterior capsule and glenohumeral ligaments probably obscure the superior shoulder portion $[18,19]$. Hidden injuries involve the biceps pulley. Disease conditions associated with pulley injuries include the following: the anterior-superior impingement, LHB tendon instability, LHB tendon lesions or tendinosis, SLAP lesions, and adhesive capsulitis $[10,20]$. In order to plan the correct therapeutic approach is important for the identification of both biceps pulley normal anatomy and biceps pulley anomalies, such as anatomic or pathologic variations [20]. When evaluating glenohumeral instability, MR arthrography ensures an appropriate joint distension and excellent anatomic detail, which improves the diagnostic value of the method $[21,22]$. However, ours is a purely descriptive study of the shoulder anatomy and its anatomical variants, we refer to the orthopaedist the decision of how to intervene correlating our information with clinical data. In a series of patients studied by Le Heuc et al. MRI, without contrast agent, usually failed in the identification of some lesions diagnosed using arthroscopy [23]. The lack of identification was explained by the need of capsular distention that gives a better view of all anatomic structures and a better contrast between the glenoid labrum, capsule, glenohumeral ligaments, biceps tendon, and articular surface of the rotator cuff $[24,25]$. However, it is important to underline that the results of Le Heuc et al. should not be considered as conclusive because the number of analysed patients was very low $(n=10)$ [23].

Magee et al. compared the sensibility and specificity of conventional MRI against MRI arthrography in 150 shoulders, and they found that the sensibility for conventional MRI for labral anterior injury detection was $83 \%$ and the specificity was $100 \%$; for the SLAP injuries the detection was $85 \%$ and the specificity was $100 \%$; and for SSP tendon lesions the detection was $92 \%$ and the specificity was $100 \%$. MRI arthrography results were as follows: $98 \%$ and $100 \%$ for labral anterior injury detection, $98 \%$ and 99\% for SLAP lesion detection, and 100\% for both sensi- 

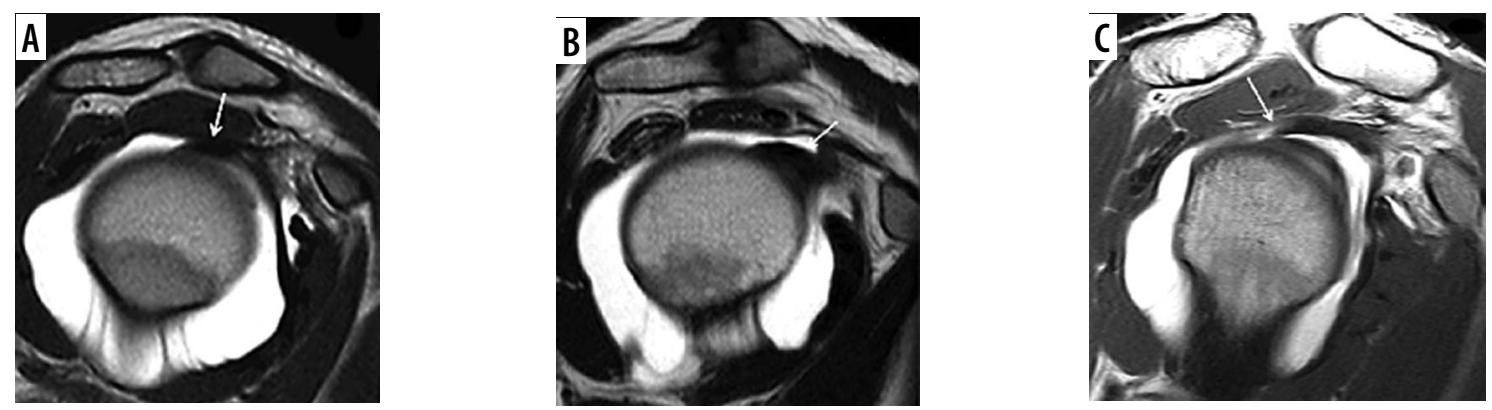

Figure 6. Magnetic resonance arthrography. SE T1w image on the oblique sagittal plane. Site of long head biceps anchor on the glenoid (arrow): at 12 o'clock (A), more anterior at $10^{\prime} \mathrm{clock}(\mathrm{B})$, and more posterior at $11 \mathrm{o}^{\prime} \mathrm{clock}(\mathrm{C})$
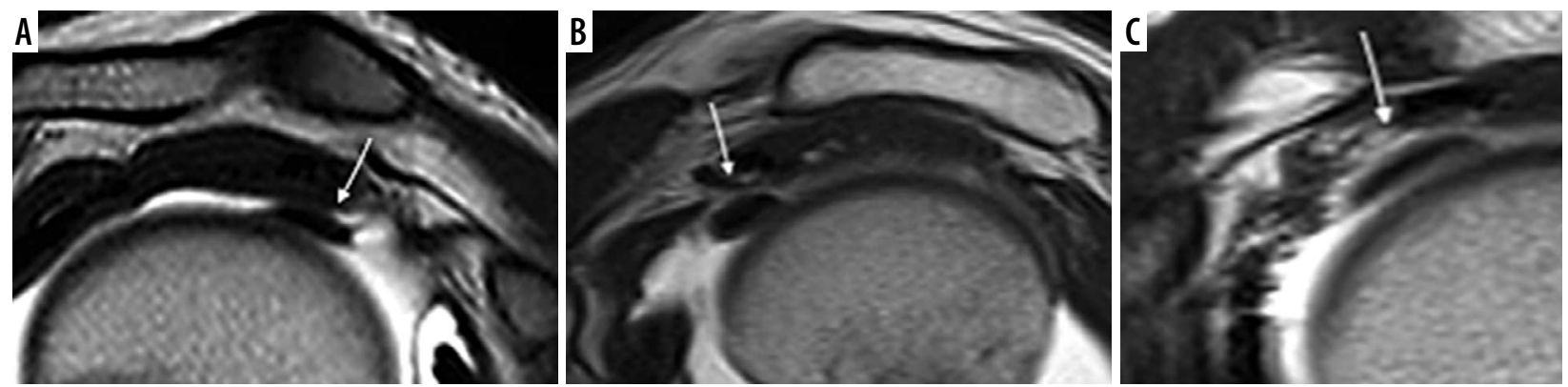

Figure 7. Magnetic resonance arthrography. SE T1w image on the oblique sagittal plane. Long head biceps tendon's thickness and shape (arrow): oval (A), rounded (B), or flat (C)

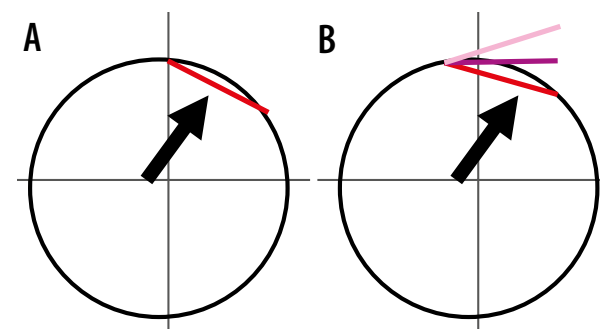

Figure 8. Schematic representation of glenoid "clockface" proposed mechanism for association between a predominantly posterior LHB attachment and posterior tears: A) A completely posterior attachment (red) effectively counteracts the vectors (black arrow) that normally promote humeral subluxation in abduction/external rotation. B) As the site of maximal vector force is fixed, with a predominantly posterior attachment, the unequal distribution of the tendon fibres over the vector site leads to partial opposing forces to humeral subluxation leading to a progressive lift off of the biceps tendon at its posterior labral attachment (posterior tears with progressive lift off indicated by lighter shades of pink)

bility and specificity for rotator cuff lesion detection [26]. These results confirmed that the diagnostic accuracy of MR arthrography of the shoulder is better than conventional MRI of the shoulder for rotator free interval diseases; MRI arthrography is now considered the gold standard in the study of the shoulder instability [1]. In addition, arthroscopic correlation with MR findings might be helpful to determine the accuracy of MR in defining anatomy, although recent studies have demonstrated increased sensitivity and specificity of MR arthrography over diagnostic arthroscopy, suggesting the superiority of capsular distention and contrast resolution $[1,27,28]$. The LHB tendon's role in the mobility and stability of glenohumeral articulation has not yet been clearly defined [29-31]. The LHB is important in glenohumeral stability, acting as a dynamic stabilizer and a depressor of the humeral head and also an elevator of the glenoid labrum [32]. Our purpose was to estimate the frequency of the LHB pulley anatomic variations; a previous study described it with arthroscopy [16]. In the literature some congenital anatomic variations of the LHB intra-articular portion are reported regarding its relationship with the rotator cuff. The majority of these studies are based on arthroscopy $[3,8,11,13,14,17,22,33-36]$; one was performed using anatomic dissection [37] while another 3 were conducted with MR-arthrography associated with arthroscopy $[15,38,39]$. Some studies described hypoplasia and/or LHB absence [2,3,14,15,17,22,36,37], while others reported cases of divisum or split tendon [13,39]. Dierickx et al. analysed 2976 shoulder arthroscopies highlighting 57 anatomic variations of the LHB tendon; they created a classification based on the anatomy of the LHB and differentiated them into 4 major families: 29 mesotenon, 15 adherent, 11 split or divisum, 2 absent [16]. In our study we found the mesotenon variant in 31 cases $(9.5 \%)$, the adherent variant in 40 cases $(12.3 \%)$, the LHB divisum in 3 cases $(0.9 \%)$, and in no cases we found absence of the tendon. We can speculate an overestimation of the adherent variant, which would take a neutral position of the limb and to an incorrect valuation of the articulation in dynamic phases.

Previous studies have demonstrated that the LHB tendon variants are congenital, a consequence of partial detachment from the mesothelium or synovial fusion with the inferior surface of the capsule $[17,40]$. By now, most of these conditions are not considered significant or responsible for shoulder disorders. However, only a few 
authors have considered these variants as a possible cause of disease in adult life despite these abnormalities being present since birth, but the results of such studies are not definitive $[13,34,35]$. Dierickx's arthroscopic study showed that partial mesotenon can cause bicepsrelated complaints. Partial lateral adhesion can cause an hourglass-type of impingement, whereas the complete adherent or solid fusion of the LHB tendon to the inferior surface of the capsule (with extension to the superior glenoid labrum) might be associated with rotator cuff lesions [16]. The LHB "divisum" (double origin biceps) has a high incidence in young patients with the framework of impingement and cuff lesions rotator, and it may be a contributory cause [16]. We found a statistically significant correlation $(p=0.007)$ between the mesotenon group and the free LHB group regarding the presence of rotator interval synovitis; in particular in the group with mesotenon the prevalence of patients with rotator interval synovitis (16.6\%) was higher than that of free LHB (7.9\%). We also found a statistically significant association between the adherent LHB group and the free LHB group regarding the presence of SLAP lesions. The SLAP lesions diagnosed in the ADH group were 15/25 (60\%) whereas in the LHB free group comprised 64/164 (39\%). According to the literature, the most frequently affects type $2[8,14,26,40]$. Wahl et al. also stated that the LHB adherent was correlated to SLAP lesion type 2 [36]. The LHB divisum, in our series, was a rare finding $(3 / 326$, $0.9 \%)$; however, we found in our 3 cases that there was not a correlation with rotator cuff lesions: a patient presented impingement associated with a SLAP lesion type 4 , one presented a SLAP lesion type 3 associated with superior glenohumeral ligament (SGHL) lesion and rotator interval synovitis, and one patient did not show superior shoulder pathology. We also considered the anatomical variations of the shoulder; these variants are almost exclusive to the superior region of the shoulder (hours 9-3) and can create some problems in the differential diagnosis of diseases, such as superior shoulder diseases and SLAP lesions $[8,22,24,25,28]$. From the analysis of our data, we have not found a statistically significant difference between the incidence of anatomical variations in the various groups analysed, except for the presence of the sublabral recess $(p=0.018)$ in the $\mathrm{ADH}$ group $(3 / 10,30 \%)$ compared with the free LHB group $(3 / 77,3.9 \%)$. To the best of our knowledge, no previous studies have investigated the correlation between anatomical variations of the LHB and anatomical variations of superior shoulder. Regarding the LHB tendon origin from the glenoid labrum, in a study of 31 cadaveric shoulders, Demondion et al. described 4 types of LHB tendon origin: in $64.5 \%$ of cases the LHB tendon was inserted mainly in the posterosuperior portion the glenoid labrum, in $19.4 \%$ the origin was in the posterior level of the glenoid labrum, in $6.4 \%$ of cases the origin was found in the supraglenoid tubercle of the scapula, and in 3 cases LHB tendon was inserted into the LHB groove [41].
Table 3. LHB tendon course anatomical variants. Frequency of long head biceps anatomic variants, based on Dierickx's arthroscopic classification 11

\begin{tabular}{|l|c|}
\hline \multicolumn{2}{|l|}{ LHB free } \\
\hline Separated to supraspinatus tendon & $252 / 326(77.30 \%)$ \\
\hline LHB adherent & $40 / 326(12.26 \%)$ \\
\hline Complete adhesion & $32 / 40(80 \%)$ \\
\hline Partial distal adhesion & $3 / 40(7.5 \%)$ \\
\hline Partial proximal adhesion & $5 / 40(12.5 \%)$ \\
\hline LHB split "divisum" & $3(0.92 \%)$ \\
\hline SPL-D0 & $31 / 326(9.50 \%)$ \\
\hline LHB mesotenon & $4 / 31(12.9 \%)$ \\
\hline MESO-PU & $1 / 31(3.2 \%)$ \\
\hline MESO-PA & $11 / 31(35.5 \%)$ \\
\hline MESO-SB & $15 / 31(48.4 \%)$ \\
\hline MESO-VI & 0 \\
\hline LHB absent &
\end{tabular}

LHB - long head biceps, MESO-PU - mesotenon pulley, MESO-PA - mesotenon partial, MESO-SB - mesotenon small-band, MESO-VI - mesotenon vinculum, SSP - supraspinatus

Vangsness et al. described 4 variants of insertion of the LHB tendon from the glenoid labrum: entirely posterior (22\%), predominantly posterior (33\%), median (37\%), and anterior (8\%) [42]. Tuoheti et al. demonstrated histologically that most of the tendon fibres are oriented towards the posterior side of the labrum and that only some fibres extend to the front portion [43]. In our study, the LHB tendon insertion on the glenoid is been seen in $242 / 326(74.2 \%)$ at 12 o'clock, in $47 / 326(14.5 \%)$ cases it was back in the $10-11$ oclock position, and in $37 / 326(11.3 \%)$ cases it was more anterior, at the 1 oclock position. Egea et al. described a case with MR and arthroscopy of LHB emerging from the glenohumeral joint capsule, without intra-articular origin; in our series no similarity was found [12].

Regarding the thickness and the shape of the LHB tendon, to our knowledge, in the literature there is only one work, by Buck et al., that analyses the position, shape, and orientation of the LHB with respect to the bicipital groove; we considered these parameters with respect to the intra-articular portion of LHB tendon [44]. Buck et al. enrolled 53 asymptomatic volunteers, and the morphological characteristics of LHB were evaluated in a neutral position in intra-rotation and external rotation of the bicipital groove at three levels (upper, middle and lower) with the following results: at the superior level, the LHB was flat, independently of the arm position; at the middle level in a neutral position the LHB was flat, while in an intrarotation position it had a "floating point" shape and was oval in external rotation; at the lower level the LHB had a mainly oval shape except during intra-rotation, where it takes more rounded shape [44]. In our series we observed in $221 / 326(67.8 \%)$ patients that, at the level of the intraarticular portion, the LHB had an oval shape with a value 
of the ratio between transverse diameters and cranio-caudal diameters from 2.51 to 4.47 ; in $17 / 326$ (5.2\%) patients the LHB had a more rounded shape (values from 1.47 to 2.5 ), and in $88 / 326$ cases (27\%) the tendon appeared thinned (with values between 4.53 and 9.75). Buck et al. also showed that the position of the LHB into the bicipital groove depends on the degree of rotation of the shoulder: in the neutral position, used in the MR-arthrography studies, the LHB is eccentric with respect to the groove [44]. In our series the LHB tendon presented this orientation in most of the cases (323/326; 99.1\%), in $2 / 326$ $(0,6 \%)$ cases we observed a sub-dislocation of the tendon; in only one patient $(0.3 \%)$ the LHB tendon was dislocated medially to the groove. The medial dislocation of the LHB tendon is a rare occurrence that is rarely described in the literature; Slatis et al. showed that in 286 anatomical dissections of the shoulder, only 4 (1.4\%) had a LHB dislocation, in all cases associated with lesions of the rotator cuff; our only case of dislocation was observed in a patient with a complete lesion of the subscapularis tendon [6]. Because so few cases have been reported in the literature, is not clear yet if the presence of LHB pulley variants might be a cause of instability of the superior shoulder or if the observed findings are to be considered isolated and meaningless. It is possible that the adherence of the LHB tendon to the inferior portion of the capsule draws up more frequently ( $p=0.05$ in the series staff) to a lesion of the superior glenoid labrum (SLAP lesions), due to the strong relationship between the fibrocartilage and biceps anchor. Regarding statistical significance $(p=0.018$ in our series) between LHB adherent and the sublabral recess, we can assume that the intimate relationship between the LHB and the SSP tendons, associated with the biceps tendon attachment of the superior labrum to the glenoid, establish, during embryonic development, to a greater tensile portion on cartilage and hence development of sublabral recess. The prevalence of rotator interval synovitis in the mesotenon group compared to the
LHB free group, with statistical significance $(p=0.007)$, cannot be supported by a pathophysiological explanation and can be considered an artifact without clinical significance. In this study there are some limitations. First of all, the lack of systematic correlation of the MR arthrography findings with the arthroscopic data. However, it our opinion that this should be considered a minor limitation because in the literature MR arthrography is considered the reference standard in the evaluation of the superior portion of the shoulder, a region that is rarely explored in arthroscopy, probably due to the anterior approach of this method [18,19,21,22,24]. Moreover, we do not have enough data to make a correlation between the anatomical variant and the clinical symptomatology. However, the complexity of the topic is supported by the fact that even the orthopaedist often does not know what clinical significance to attribute to these anatomical variants.

\section{Conclusions}

The results of our study suggest that MR arthrography of the shoulder is the most accurate established imaging method for demonstrating abnormalities of the glenoid labrum and associated structures. Knowledge of the existence of LHB intra-articular portion anatomical variants and the description of their MR arthrography findings are essential to avoid errors in the differential diagnosis of superior shoulder anatomical variants and diseases. However, the role of LHB anatomical variants in the function of the shoulder is still unclear, thus further studies with a larger cohort of patients and with a longer follow-up will be necessary to validate the association of anatomical variants and shoulder instability.

\section{Conflict of interest}

The authors report no conflict of interest.

\section{References}

1. Jakanani GC, Botchu R, Rennie WJ. The MR arthrographic anatomy of the biceps labral insertion and its morphological significance with labral tears in patients with shoulder instability. Eur J Radiol 2012; 81: 3390-3393.

2. Ghalayini SRA, Board TN, Srinivasan MS. Anatomic variations in the long head of biceps: contribution to shoulder dysfunction. Arthroscopy 2007; 23: 1012-1018.

3. Glueck DA, Mair SD, Johnson DL. Shoulder instability with absence of the long head of the biceps tendon. Arthroscopy 2003; 19: 787789.

4. Harryman DT, Sidles JA, Harris SL, Matsen FA. The role of the rotator interval capsule in passive motion and stability of the shoulder. J Bone Joint Surg Am 1992; 74: 53-66.
5. Bennett WF. Subscapularis, medial, and lateral head coracohumeral ligament insertion anatomy. Arthroscopic appearance and incidence of 'hidden' rotator interval lesions. Arthroscopy 2001; 17: 173-180.

6. Slätis P, Aalto K. Medial dislocation of the tendon of the long head of the biceps brachii. Acta Orthop Scand 1979; 50: 73-77.

7. Jeong JY, Park SM, Park YE, Yoo JC. Morphological classification of anatomical variants of the intra-articular portion of the long head of the biceps brachii tendon and analysis of the incidence and the relationship with shoulder disease for each subtype. J Orthop Surg Hong Kong 2017; 25: 2309499017742207.

8. Genovese E, Spanò E, Castagna A, et al. MR-arthrography in superior instability of the shoulder: correlation with arthroscopy. Radiol Med (Torino) 2013; 118: 1022-1033. 
9. Sher JS, Iannotti JP, Williams GR, et al. The effect of shoulder magnetic resonance imaging on clinical decision making. J Shoulder Elbow Surg 1998; 7: 205-209.

10. Barile A, Lanni G, Conti L, et al. Lesions of the biceps pulley as cause of anterosuperior impingement of the shoulder in the athlete: potentials and limits of MR arthrography compared with arthroscopy. Radiol Med (Torino) 2013; 118: 112-122.

11. Bennett WF. Visualization of the anatomy of the rotator interval and bicipital sheath. Arthroscopy 2001; 17: 107-111.

12. Egea JM, Melguizo C, Prados J, Aránega A. Capsular origin of the long head of the biceps brachii tendon: a clinical case. Rom J Morphol Embryol 2010; 51: 375-377.

13. Enad JG. Bifurcate origin of the long head of the biceps tendon. Arthroscopy 2004; 20: 1081-1083.

14. Franco JC, Knapp TP, Mandelbaum BR. Congenital absence of the long head of the biceps tendon. A case report. J Bone Joint Surg Am 2005; 87: 1584-1586.

15. Gaskin CM, Golish SR, Blount KJ, Diduch DR. Anomalies of the long head of the biceps brachii tendon: clinical significance, MR arthrographic findings, and arthroscopic correlation in two patients. Skeletal Radiol 2007; 36: 785-789.

16. Dierickx C, Ceccarelli E, Conti M, Vanlommel J, Castagna A. Variations of the intra-articular portion of the long head of the biceps tendon: a classification of embryologically explained variations. J Shoulder Elbow Surg 2009; 18: 556-565.

17. Audenaert EA, Barbaix EJ, Van Hoonacker P, Berghs BM. Extraarticular variants of the long head of the biceps brachii: a reminder of embryology. J Shoulder Elbow Surg 2008; 17 (1 Suppl): 114S-117S.

18. Walch G, Nove-Josserand L, Levigne C, Renaud E. Tears of the supraspinatus tendon associated with 'hidden' lesions of the rotator interval. J Shoulder Elbow Surg 1994; 3: 353-360.

19. Chung CB, Dwek JR, Cho GJ, Lektrakul N, Trudell D, Resnick D Rotator cuff interval: evaluation with MR imaging and MR arthrography of the shoulder in 32 cadavers. J Comput Assist Tomogr 2000; 24: 738-743.

20. Nakata W, Katou S, Fujita A, Nakata M, Lefor AT, Sugimoto H. Biceps pulley: normal anatomy and associated lesions at MR arthrography. Radiographics 2011; 31: 791-810.

21. Jee WH, McCauley TR, Katz LD, Matheny JM, Ruwe PA, Daigneault JP. Superior labral anterior posterior (SLAP) lesions of the glenoid labrum: reliability and accuracy of MR arthrography for diagnosis. Radiology 2001; 218: 127-132.

22. Smith DK, Chopp TM, Aufdemorte TB, Witkowski EG, Jones RC. Sublabral recess of the superior glenoid labrum: study of cadavers with conventional nonenhanced MR imaging, MR arthrography, anatomic dissection, and limited histologic examination. Radiology 1996; 201: 251-256.

23. Le Huec JC, Schaeverbeke T, Moinard M, et al. Traumatic tear of the rotator interval. J Shoulder Elbow Surg 1996; 5: 41-46.

24. Beltran J, Bencardino J, Mellado J, Rosenberg ZS, Irish RD. MR arthrography of the shoulder: variants and pitfalls. Radiographics 1997; 17: 1403-1412; discussion 1412-1415

25. Kreitner KF, Botchen K, Rude J, Bittinger F, Krummenauer F, Thelen M. Superior labrum and labral-bicipital complex: MR imaging with pathologic-anatomic and histologic correlation. AJR Am J Roentgenol 1998; 170: 599-605.
26. Magee T. 3-T MRI of the shoulder: is MR arthrography necessary? AJR Am J Roentgenol 2009; 192: 86-92.

27. Amin MF, Youssef AO. The diagnostic value of magnetic resonance arthrography of the shoulder in detection and grading of SLAP lesions: comparison with arthroscopic findings. Eur J Radiol 2012; 81: 2343-2347.

28. Chang D, Mohana-Borges A, Borso M, Chung CB. SLAP lesions: anatomy, clinical presentation, MR imaging diagnosis and characterization. Eur J Radiol 2008; 68: 72-87.

29. Iannotti JP, Williams GR. Disorders of the Shoulder: Diagnosis \& Management. Lippincott Williams \& Wilkins; 2007; p. 762.

30. Kido T, Itoi E, Konno N, Sano A, Urayama M, Sato K. The depressor function of biceps on the head of the humerus in shoulders with tears of the rotator cuff. J Bone Joint Surg Br 2000; 82: 416-419.

31. Yamaguchi K, Riew KD, Galatz LM, Syme JA, Neviaser RJ. Biceps activity during shoulder motion: an electromyographic analysis. Clin Orthop Relat Res 1997; 336: 122-129.

32. Gramstad GG, Sears BW, Marra G. Variation of tension in the long head of the biceps tendon as a function of limb position with simulated biceps contraction. Int J Shoulder Surg 2010; 4: 8-14.

33. Hyman JL, Warren RF. Extra-articular origin of biceps brachii. Arthroscopy 2001; 17: E29.

34. MacDonald PB. Congenital anomaly of the biceps tendon and anatomy within the shoulder joint. Arthroscopy 1998; 14: 741-742.

35. Ogawa K, Naniwa T. A rare variation of the biceps: a possible cause of degeneration of the rotator cuff. J Shoulder Elbow Surg 1998; 7: 295 297.

36. Wahl CJ, MacGillivray JD. Three congenital variations in the long head of the biceps tendon: a review of pathoanatomic considerations and case reports. J Shoulder Elbow Surg 2007; 16: e25-30.

37. Georgiev GP, Jelev L. Bilateral hypoplasia of the long head of the biceps brachii muscle. Int J Shoulder Surg 2011; 5: 26-27.

38. Gheno R, Zoner CS, Buck FM, et al. Accessory head of biceps brachii muscle: anatomy, histology, and MRI in cadavers. AJR Am J Roentgenol 2010; 194: W80-83.

39. Kim KC, Rhee KJ, Shin HD. A long head of the biceps tendon confluent with the intra-articular rotator cuff: arthroscopic and MR arthrographic findings. Arch Orthop Trauma Surg 2009; 129: 311-314.

40. Herold T, Hente R, Zorger N, et al. Indirect MR-arthrography of the shoulder-value in the detection of SLAP-lesions. Rofo 2003; 175: 1508-1514 [Article in German].

41. Demondion X, Maynou C, Van Cortenbosch B, Klein K, Leroy X, Mestdagh $\mathrm{H}$. Relationship between the tendon of the long head of the biceps brachii muscle and the glenoid labrum. Morphologie 2001; 85: 5-8 [Article in French].

42. Vangsness CT, Jorgenson SS, Watson T, Johnson DL. The origin of the long head of the biceps from the scapula and glenoid labrum. An anatomical study of 100 shoulders. J Bone Joint Surg Br 1994; 76: 951-954.

43. Tuoheti $\mathrm{Y}$, Itoi E, Minagawa $\mathrm{H}$, et al. Attachment types of the long head of the biceps tendon to the glenoid labrum and their relationships with the glenohumeral ligaments. Arthroscopy 2005; 21: 1242-1249.

44. Buck FM, Dietrich TJ, Resnick D, Jost B, Pfirrmann CWA. Long biceps tendon: normal position, shape, and orientation in its groove in neutral position and external and internal rotation. Radiology 2011; 261: 872-881. 